International Mathematical Forum, Vol. 8, 2013, no. 31, 1539 - 1551

HIKARI Ltd, www.m-hikari.com

http://dx.doi.org/10.12988/imf.2013.37135

\title{
The Best Possible Lehmer Mean Bounds for a Convex Combination of Logarithmic and Harmonic Means ${ }^{1}$
}

\author{
Zhijun Guo \\ School of Mathematics and Computation Science \\ Hunan City University \\ Yiyang, Hunan, 413000, P. R. China \\ Xuhui Shen \\ College of Nursing, Huzhou Teachers College \\ Zhejiang, Huzhou, 313000, P.R. China \\ Yuming Chu \\ College of Mathematics and Computation Science \\ Hunan City University \\ Yiyang, Hunan, 413000, P.R. China \\ Corresponding author. e-mail: chuyuming@hutc.zj.cn
}

Copyright (c) 2013 Zhijun Guo, Xuhui Shen and Yuming Chu. This is an open access article distributed under the Creative Commons Attribution License, which permits unrestricted use, distribution, and reproduction in any medium, provided the original work is properly cited.

\footnotetext{
Abstract

For $r \in \mathbb{R}$ and $a, b>0$ the Lehmer mean $L_{r}(a, b)$, logarithmic mean $L(a, b)$, and harmonic mean $H(a, b)$ are defined by

$$
L_{r}(a, b)=\frac{a^{r+1}+b^{r+1}}{a^{r}+b^{r}}, \quad L(a, b)= \begin{cases}\frac{b-a}{\log b-\log a}, & b \neq a, \\ a, & b=a,\end{cases}
$$

${ }^{1}$ This research was supported by the Natural Science Foundation of China (Grant Nos. 11071069 and 11171307), the Natural Science Foundation of Zhejiang Province (Grant Nos. LY13H070004 and LY13A010004), and the Natural Science Foundation of the Department of Education of Hunan Province (Grant No. 12C0577).
} 
and $H(a, b)=\frac{2 a b}{a+b}$, respectively. In this paper, we answer the question: For $\alpha \in(0,1)$, what are the largest value $p$ and least value $q$ such that the double inequality $L_{q}(a, b)>\alpha H(a, b)+(1-\alpha) L(a, b)>L_{p}(a, b)$ holds for all $a, b>0$ with $a \neq b$ ?

\section{Mathematics Subject Classification: 26E60}

Keywords: Lehmer mean, logarithmic mean, harmonic mean

\section{Introduction}

For $r \in \mathbb{R}$ and $a, b>0$ the Lehmer mean $L_{r}(a, b)$, logarithmic mean $L(a, b)$, and harmonic mean $H(a, b)$ are defined by

$$
\begin{gathered}
L_{r}(a, b)=\frac{a^{r+1}+b^{r+1}}{a^{r}+b^{r}}, \\
L(a, b)= \begin{cases}\frac{b-a}{\log b-\log a}, & b \neq a, \\
a, & b=a,\end{cases}
\end{gathered}
$$

and

$$
H(a, b)=\frac{2 a b}{a+b}
$$

It is well-known that $L_{r}(a, b)$ is continuous and strictly increasing with respect to $r \in \mathbb{R}$ for fixed $a$ and $b$ with $a \neq b$. In the recent past, the Lehmer mean has been attracted the attention of many mathematicians [1-14]. Many means are the special cases of the Lehmer mean, for example,

$$
\begin{aligned}
& A(a, b)=\frac{a+b}{2}=L_{0}(a, b) \text { is the arithmetic mean, } \\
& G(a, b)=\sqrt{a b}=L_{-\frac{1}{2}}(a, b) \text { is the geometric mean, } \\
& H(a, b)=\frac{2 a b}{a+b}=L_{-1}(a, b) \text { is the harmonic mean. }
\end{aligned}
$$

Recently, the logarithmic mean has been the subject of intensive research. In particular, many remarkable inequalities for the logarithmic mean can be found in literatures [15-30]. It might be surprising that the logarithmic mean has applications in physics [31], economics [32], and even in meteorology [33]. In [31], the authors study a variant of Jensen's function involving logarithmic mean, which appears in a heat conduction problem. For $p \in \mathbb{R}$, let

$$
M_{p}(a, b)=\left\{\begin{array}{ll}
\left(\frac{a^{p}+b^{p}}{2}\right)^{\frac{1}{p}}, & p \neq 0, \\
\sqrt{a b}, & p=0
\end{array} \quad \text { and } \quad I(a, b)= \begin{cases}\frac{1}{e}\left(\frac{b^{b}}{a^{a}}\right)^{\frac{1}{b-a}}, & b \neq a \\
a, & b=a\end{cases}\right.
$$


be the $p$ th power mean and identric mean of two positive real numbers $a$ and $b$, respectively. Then it is well known that

$$
\begin{aligned}
& \min \{a, b\}<H(a, b)=L_{-1}(a, b)=M_{-1}(a, b)<G(a, b) \\
& =L_{-\frac{1}{2}}(a, b)=M_{0}(a, b)<L(a, b)<I(a, b)<A(a, b) \\
& =L_{0}(a, b)=M_{1}(a, b)<\max \{a, b\}
\end{aligned}
$$

for all $a, b>0$ with $a \neq b$.

In [13], Alzer presented the following sharp upper and lower Lehmer mean bounds for identric mean $I(a, b)$ :

$$
L_{-\frac{1}{6}}(a, b)<I(a, b)<L_{0}(a, b)
$$

for all $a, b>0$ with $a \neq b$.

Stolarsky [12] established that

$$
M_{2 n+1}(a, b) \leq L_{n}(a, b)
$$

for all $a, b>0$ with $a \neq b$.

In $[24,34,35]$, the authors presented bounds for $L$ and $I$ in terms of $G$ and $A$ as follows:

$$
G^{\frac{2}{3}}(a, b) A^{\frac{1}{3}}(a, b)<L(a, b)<\frac{2}{3} G(a, b)+\frac{1}{3} A(a, b)
$$

and

$$
I(a, b)>\frac{1}{3} G(a, b)+\frac{2}{3} A(a, b)
$$

for all $a, b>0$ with $a \neq b$.

The following inequalities can be found in [26]:

$$
G^{\frac{1}{2}}(a, b) A^{\frac{1}{2}}(a, b)<L^{\frac{1}{2}}(a, b) I^{\frac{1}{2}}(a, b)<\frac{1}{2}(L(a, b)+I(a, b))<\frac{1}{2}(G(a, b)+A(a, b)) .
$$

The following sharp bounds for $L, I,(I L)^{\frac{1}{2}}$, and $\frac{1}{2}(I+L)$ in terms of power means are proved in [25-27, 36-39]:

$$
\begin{gathered}
M_{0}(a, b)<L(a, b)<M_{\frac{1}{3}}(a, b), \quad M_{\frac{2}{3}}(a, b)<I(a, b)<M_{\log 2}(a, b), \\
M_{0}(a, b)<\sqrt{I(a, b) L(a, b)}<M_{\frac{1}{2}}(a, b) \quad \text { and } \frac{1}{2}(I(a, b)+L(a, b))<M_{\frac{1}{2}}(a, b)
\end{gathered}
$$

for all $a, b>0$ with $a \neq b$, and the given parameters are best possible.

Alzer and Qiu [30] proved that $M_{c}(a, b)<\frac{1}{2}(L(a, b)+I(a, b))$ for all $a, b>0$ with $a \neq b$ if and only if $c \leq \log 2 /(1+\log 2)$.

The purpose of this paper is to answer the question: For $\alpha \in(0,1)$, what are the largest value $p$ and least value $q$ such that the double inequality $L_{q}(a, b)>$ 
$\alpha H(a, b)+(1-\alpha) L(a, b)>L_{p}(a, b)$ holds for all $a, b>0$ with $a \neq b$.

\section{Lemmas}

In order to prove our main result, we need several lemmas which we present in this section.

Lemma 2.1. Suppose that $\alpha \in(0,1)$ and $x \in(1, \infty)$. If $p=-\frac{2 \alpha+1}{3}$, then

$$
x^{p+2}+(1-2 \alpha) x^{p+1}+(1-2 \alpha) x+1>0 .
$$

Proof. Let $h(x)=x^{p+2}+(1-2 \alpha) x^{p+1}+(1-2 \alpha) x+1$, then simple computations lead to

$$
\begin{gathered}
\lim _{x \rightarrow 1} h(x)=4(1-\alpha)>0, \\
h^{\prime}(x)=(p+2) x^{p+1}+(1-2 \alpha)(p+1) x^{p}+1-2 \alpha, \\
\lim _{x \rightarrow 1} h^{\prime}(x)=2(p+2)(1-\alpha)>0, \\
h^{\prime \prime}(x)=(p+1) x^{p-1} h_{1}(x),
\end{gathered}
$$

where $h_{1}(x)=(p+2) x+(1-2 \alpha) p$.

Note that

$$
\begin{gathered}
\lim _{x \rightarrow 1} h_{1}(x)=\alpha^{2}+1+\frac{1}{3}(\alpha-1)^{2}>0, \\
h_{1}^{\prime}(x)=p+2>0 .
\end{gathered}
$$

Therefore, inequality (2.1) follows from (2.2)-(2.7).

Lemma 2.2. If $\alpha \in(0,1)$, then $736 \alpha^{6}+1064 \alpha^{5}-908 \alpha^{4}+2338 \alpha^{3}-5111 \alpha^{2}+$ $3665 \alpha+565>0$.

Proof. We clearly see that

$$
\begin{aligned}
& 736 \alpha^{6}+1064 \alpha^{5}-908 \alpha^{4}+2338 \alpha^{3}-5111 \alpha^{2}+3665 \alpha+565 \\
= & \alpha^{2}\left(736 \alpha^{4}-908 \alpha^{2}+281\right)+1064 \alpha^{5}+2338 \alpha^{3}-5392 \alpha^{2} \\
& +3665 \alpha+565 \\
> & \alpha^{2}\left(736 \alpha^{4}-908 \alpha^{2}+281\right)+2338 \alpha^{3}-5392 \alpha^{2}+3665 \alpha+565 \alpha \\
= & \alpha^{2}\left(736 \alpha^{4}-908 \alpha^{2}+281\right)+\alpha\left(2338 \alpha^{2}-5392 \alpha+4230\right) .
\end{aligned}
$$

The discriminants $\Delta_{1}$ and $\Delta_{2}$ of the quadratic functions $736 t^{2}-908 t+281$ and $2338 t^{2}-5392 t+4320$ satisfy

$$
\Delta_{1}=(-908)^{2}-4 \times 736 \times 281=-2800<0
$$

and

$$
\Delta_{2}=(-5392)^{2}-4 \times 2338 \times 4230=-10485296<0
$$

respectively. Therefore, Lemma 2.2 follows from (2.8)-(2.10). 
Lemma 2.3. If $\alpha \in(0,1)$, then $808 \alpha^{5}+2836 \alpha^{4}+1750 \alpha^{3}+1343 \alpha^{2}-3124 \alpha+$ $1085>0$.

Proof. We clearly see that

$$
\begin{aligned}
& 808 \alpha^{5}+2836 \alpha^{4}+1750 \alpha^{3}+1343 \alpha^{2}-3124 \alpha+1085 \\
> & 1750 \alpha^{3}+1350 \alpha^{2}-3150 \alpha+1050 \\
= & 50\left(35 \alpha^{3}+27 \alpha^{2}-63 \alpha+21\right) .
\end{aligned}
$$

It is not difficult to verify that

$$
\begin{aligned}
& \min _{\alpha \in(0,1)}\left(35 \alpha^{3}+27 \alpha^{2}-63 \alpha+21\right) \\
= & 35\left(\frac{4 \sqrt{51}-9}{35}\right)^{3}+27\left(\frac{4 \sqrt{51}-9}{35}\right)^{2}-63\left(\frac{4 \sqrt{51}-9}{35}\right)+21 \\
= & 0.333 \ldots>0 .
\end{aligned}
$$

Therefore, Lemma 2.3 follows from (2.11) and (2.12).

Lemma 2.4. If $\alpha \in(0,1)$, then $304 \alpha^{5}+724 \alpha^{4}-488 \alpha^{3}+839 \alpha^{2}-3361 \alpha+2387>$ 0 .

Proof. We clearly see that

$$
\begin{aligned}
& 304 \alpha^{5}+724 \alpha^{4}-488 \alpha^{3}+839 \alpha^{2}-3361 \alpha+2387 \\
> & 724 \alpha^{4}-488 \alpha^{3}+488 \alpha^{2}+351 \alpha^{2}-3361 \alpha+2387 \\
> & 724 \alpha^{4}+351 \alpha^{4}-3361 \alpha+2387 \\
= & 1075 \alpha^{4}-3361 \alpha+2387
\end{aligned}
$$

It is easy to verify that

$$
\begin{aligned}
& \min _{\alpha \in(0,1)}\left(1075 \alpha^{4}-3361 \alpha+2387\right) \\
= & 1075\left(\sqrt[3]{\frac{3361}{4300}}\right)^{4}-3361\left(\sqrt[3]{\frac{3361}{4300}}\right)+2387 \\
= & 64.995 \ldots>0
\end{aligned}
$$

Therefore, Lemma 2.4 follows from (2.13) and (2.14).

\section{Main Result}

Theorem 3.1. If $\alpha \in(0,1)$, then $L_{0}(a, b)>\alpha H(a, b)+(1-\alpha) L(a, b)>$ $L_{-\frac{2 \alpha+1}{3}}(a, b)$ for all $a, b>0$ with $a \neq b$, and $L_{0}(a, b)$ and $L_{-\frac{2 \alpha+1}{3}}(a, b)$ are the best possible upper and lower Lehmer mean bounds for the sum $\alpha H(a, b)+$ $(1-\alpha) L(a, b)$.

Proof. Without loss of generality, we assume that $a>b$. From (1.4) we clearly see that $L_{0}(a, b)>\alpha H(a, b)+(1-\alpha) L(a, b)$.

Next, we prove that

$$
\alpha H(a, b)+(1-\alpha) L(a, b)>L_{-\frac{2 \alpha+1}{3}}(a, b) .
$$


Let $x=\frac{a}{b}>1$ and $p=-\frac{2 \alpha+1}{3}$, then (1.1)-(1.3) lead to

$$
\begin{aligned}
& \alpha H(a, b)+(1-\alpha) L(a, b)-L_{p}(a, b) \\
= & \frac{b\left[x^{p+2}+(1-2 \alpha) x^{p+1}+(1-2 \alpha) x+1\right]}{(1+x)\left(1+x^{p}\right) \log x} f(x),
\end{aligned}
$$

where $f(x)=\frac{(1-\alpha)\left(x^{p+2}-x^{p}+x^{2}-1\right)}{x^{p+2}+(1-2 \alpha) x^{p+1}+(1-2 \alpha) x+1}-\log x$. Note that

$$
\begin{gathered}
\lim _{x \rightarrow 1} f(x)=0, \\
f^{\prime}(x)=\frac{f_{1}(x)}{x\left[x^{p+2}+(1-2 \alpha) x^{p+1}+(1-2 \alpha) x+1\right]^{2}},
\end{gathered}
$$

where $f_{1}(x)=-x^{2 p+4}+\left(2 \alpha^{2}+\alpha-1\right) x^{2 p+3}-\left(4 \alpha^{2}-2 \alpha-1\right) x^{2 p+2}+\left(2 \alpha^{2}-3 \alpha+\right.$ 1) $x^{2 p+1}-p(1-\alpha) x^{p+4}+2 \alpha(2 \alpha-1) x^{p+3}-2\left(4 \alpha^{2}-2 \alpha+\alpha p-p\right) x^{p+2}+2 \alpha(2 \alpha-$ 1) $x^{p+1}-p(1-\alpha) x^{p}+\left(2 \alpha^{2}-3 \alpha+1\right) x^{3}-\left(4 \alpha^{2}-2 \alpha-1\right) x^{2}+\left(2 \alpha^{2}+\alpha-1\right) x-1$,

$$
\lim _{x \rightarrow 1} f_{1}(x)=0
$$

Let $f_{2}(x)=x^{4-p} f_{1}^{(4)}(x), f_{3}(x)=\frac{1}{2(p+1)} f_{2}^{\prime}(x), f_{4}(x)=\frac{1}{2} f_{3}^{\prime}(x), f_{5}(x)=$ $x^{4-p} f_{4}^{\prime \prime \prime}(x), f_{6}(x)=\frac{1}{(p+1)(p+2)} f_{5}^{\prime}(x)$, and $f_{7}(x)=\frac{1}{2(p+3)(2 p+3)} f_{6}^{\prime}(x)$, then simple computations yield that

$$
\begin{aligned}
& f_{1}^{\prime}(x)=-2(p+2) x^{2 p+3}+(2 p+3)\left(2 \alpha^{2}+\alpha-1\right) x^{2 p+2}-2(p+1) \\
& \times\left(4 \alpha^{2}-2 \alpha-1\right) x^{2 p+1}+(2 p+1)\left(2 \alpha^{2}-3 \alpha+1\right) x^{2 p} \\
&-p(p+4)(1-\alpha) x^{p+3}+2 \alpha(p+3)(2 \alpha-1) x^{p+2}-2(p+2) \\
& \times\left(4 \alpha^{2}-2 \alpha+\alpha p-p\right) x^{p+1}+2 \alpha(p+1)(2 \alpha-1) x^{p} \\
&-p^{2}(1-\alpha) x^{p-1}+3\left(2 \alpha^{2}-3 \alpha+1\right) x^{2}-2\left(4 \alpha^{2}-2 \alpha-1\right) x \\
&+2 \alpha^{2}+\alpha-1, \\
& \quad \lim _{x \rightarrow 1} f_{1}^{\prime}(x)=0 \\
& f_{1}^{\prime \prime}(x)=-2(p+2)(2 p+3) x^{2 p+2}+2(p+1)(2 p+3)\left(2 \alpha^{2}+\alpha-1\right) x^{2 p+1} \\
&-2(p+1)(2 p+1)\left(4 \alpha^{2}-2 \alpha-1\right) x^{2 p}+2 p(2 p+1) \\
& \times\left(2 \alpha^{2}-3 \alpha+1\right) x^{2 p-1}-p(p+3)(p+4)(1-\alpha) x^{p+2} \\
&+2 \alpha(p+2)(p+3)(2 \alpha-1) x^{p+1}-2(p+1)(p+2) \\
& \times\left(4 \alpha^{2}-2 \alpha+\alpha p-p\right) x^{p}+2 \alpha p(p+1)(2 \alpha-1) x^{p-1} \\
&-p^{2}(p-1)(1-\alpha) x^{p-2}+6\left(2 \alpha^{2}-3 \alpha+1\right) x \\
&-2\left(4 \alpha^{2}-2 \alpha-1\right), \\
& \quad \lim _{x \rightarrow 1} f_{1}^{\prime \prime}(x)=0
\end{aligned}
$$




$$
\begin{aligned}
& f_{1}^{\prime \prime \prime}(x)=-4(p+1)(p+2)(2 p+3) x^{2 p+1}+2(p+1)(2 p+1)(2 p+3) \\
& \times\left(2 \alpha^{2}+\alpha-1\right) x^{2 p}-4 p(p+1)(2 p+1)\left(4 \alpha^{2}-2 \alpha-1\right) x^{2 p-1} \\
& +2 p(2 p-1)(2 p+1)\left(2 \alpha^{2}-3 \alpha+1\right) x^{2 p-2}-p(p+2)(p+3) \\
& \times(p+4)(1-\alpha) x^{p+1}+2 \alpha(p+1)(p+2)(p+3)(2 \alpha-1) x^{p} \\
& -2 p(p+1)(p+2)\left(4 \alpha^{2}-2 \alpha+\alpha p-p\right) x^{p-1}+2 \alpha p(p-1) \\
& \times(p+1)(2 \alpha-1) x^{p-2}-p^{2}(p-1)(p-2)(1-\alpha) x^{p-3} \\
& +6\left(2 \alpha^{2}-3 \alpha+1\right) \text {, } \\
& \lim _{x \rightarrow 1} f_{1}^{\prime \prime \prime}(x)=0, \\
& f_{2}(x)=-4(p+1)(p+2)(2 p+1)(2 p+3) x^{p+4}+4 p(p+1)(2 p+1) \\
& \times(2 p+3)\left(2 \alpha^{2}+\alpha-1\right) x^{p+3}-4 p(p+1)(2 p-1)(2 p+1) \\
& \times\left(4 \alpha^{2}-2 \alpha-1\right) x^{p+2}+4 p(p-1)(2 p-1)(2 p+1) \\
& \times\left(2 \alpha^{2}-3 \alpha+1\right) x^{p+1}-p(p+1)(p+2)(p+3)(p+4)(1-\alpha) x^{4} \\
& +2 \alpha p(p+1)(p+2)(p+3)(2 \alpha-1) x^{3}-2 p(p-1)(p+1)(p+2) \\
& \times\left(4 \alpha^{2}-2 \alpha+\alpha p-p\right) x^{2}+2 \alpha p(p-1)(p-2)(p+1)(2 \alpha-1) x \\
& -p^{2}(p-1)(p-2)(p-3)(1-\alpha) \text {, } \\
& \lim _{x \rightarrow 1} f_{2}(x)=\frac{32(1-\alpha)^{2}\left(10 \alpha^{2}+25 \alpha+1\right)}{27}>0, \\
& f_{3}(x)=-2(p+2)(p+4)(2 p+1)(2 p+3) x^{p+3}+2 p(p+3)(2 p+1) \\
& \times(2 p+3)\left(2 \alpha^{2}+\alpha-1\right) x^{p+2}-2 p(p+2)(2 p-1)(2 p+1) \\
& \times\left(4 \alpha^{2}-2 \alpha-1\right) x^{p+1}+2 p(p-1)(2 p-1)(2 p+1) \\
& \times\left(2 \alpha^{2}-3 \alpha+1\right) x^{p}-2 p(p+2)(p+3)(p+4)(1-\alpha) x^{3} \\
& +3 \alpha p(p+2)(p+3)(2 \alpha-1) x^{2}-2 p(p-1)(p+2) \\
& \times\left(4 \alpha^{2}-2 \alpha+\alpha p-p\right) x+\alpha p(p-1)(p-2)(2 \alpha-1), \\
& \lim _{x \rightarrow 1} f_{3}(x)=\frac{64(1-\alpha)^{2}\left(10 \alpha^{2}+25 \alpha+1\right)}{27}>0, \\
& f_{4}(x)=-(p+2)(p+3)(p+4)(2 p+1)(2 p+3) x^{p+2}+p(p+2)(p+3) \\
& \times(2 p+1)(2 p+3)\left(2 \alpha^{2}+\alpha-1\right) x^{p+1}-p(p+1)(p+2)(2 p-1) \\
& \times(2 p+1)\left(4 \alpha^{2}-2 \alpha-1\right) x^{p}+p^{2}(p-1)(2 p-1)(2 p+1) \\
& \times\left(2 \alpha^{2}-3 \alpha+1\right) x^{p-1}-3 p(p+2)(p+3)(p+4)(1-\alpha) x^{2} \\
& +3 \alpha p(p+2)(p+3)(2 \alpha-1) x-p(p-1)(p+2) \\
& \times\left(4 \alpha^{2}-2 \alpha+\alpha p-p\right) \text {, } \\
& \lim _{x \rightarrow 1} f_{4}(x)=\frac{2}{27}(1-\alpha)\left[248 \alpha^{4}+148 \alpha^{3}+786 \alpha(1-\alpha)+241 \alpha+65\right]>0, \\
& f_{4}^{\prime}(x)=-(p+2)^{2}(p+3)(p+4)(2 p+1)(2 p+3) x^{p+1}+p(p+1)(p+2) \\
& \times(p+3)(2 p+1)(2 p+3)\left(2 \alpha^{2}+\alpha-1\right) x^{p}-p^{2}(p+1)(p+2) \\
& \times(2 p-1)(2 p+1)\left(4 \alpha^{2}-2 \alpha-1\right) x^{p-1}+p^{2}(p-1)^{2}(2 p-1) \\
& \times(2 p+1)\left(2 \alpha^{2}-3 \alpha+1\right) x^{p-2}-6 p(p+2)(p+3)(p+4) \\
& \times(1-\alpha) x+3 \alpha p(p+2)(p+3)(2 \alpha-1) \text {, }
\end{aligned}
$$




$$
\begin{aligned}
\lim _{x \rightarrow 1} f_{4}^{\prime}(x)= & \frac{2}{243}(1-\alpha)\left[\alpha(1-\alpha)\left(2752 \alpha^{3}+4040 \alpha^{2}+14978\right)\right. \\
& \left.+2748 \alpha^{3}+2105 \alpha+1465\right] \\
& \quad 0
\end{aligned}
$$

We divide the proof of inequality (3.1) into two cases.

Case 1. If $\alpha \in\left(0, \frac{1}{4}\right]$, then from Lemmas 2.2-2.4 and (3.13) together with (3.15)-(3.18) we clearly see that

$$
\begin{aligned}
& \lim _{x \rightarrow 1} f_{4}{ }^{\prime \prime}(x)>0, \\
& \lim _{x \rightarrow 1} f_{5}(x)>0,
\end{aligned}
$$




$$
\begin{gathered}
\lim _{x \rightarrow 1} f_{6}(x)>0, \\
\lim _{x \rightarrow 1} f_{7}(x)>0, \\
f_{7}^{\prime}(x)>0 .
\end{gathered}
$$

It easily follows from (3.3)-(3.12) and (3.19)-(3.23) that

$$
f(x)>0
$$

for $x>1$.

Therefore inequality (3.1) follows from (3.2) and (3.24) together with Lemma 2.1.

Case 2. If $\alpha \in\left(\frac{1}{4}, 1\right)$, then from Lemmas 2.2-2.4 and (3.13) together with (3.15)-(3.18) we clearly see that (3.19) again holds and

$$
\begin{gathered}
\lim _{x \rightarrow 1} f_{5}(x)<0, \\
\lim _{x \rightarrow 1} f_{6}(x)<0, \\
\lim _{x \rightarrow 1} f_{7}(x)<0, \\
f_{7}^{\prime}(x)<0 .
\end{gathered}
$$

Inequalities (3.25)-(3.28) imply that $f_{4}^{\prime \prime}(x)$ is strictly decreasing in $(1, \infty)$. Then from (3.14) and (3.19) together with the monotonicity of $f_{4}^{\prime \prime}(x)$ we know that

$$
f_{4}^{\prime \prime}(x)>0
$$

for $x \in(1, \infty)$.

Therefore, inequality (3.1) follows from (3.2)-(3.12) and (3.29) together with Lemma 2.1.

Finally, we prove that $L_{0}(a, b)$ and $L_{-\frac{2 \alpha+1}{3}}(a, b)$ are the best possible upper and lower Lehmer mean bounds for the sum $\alpha H(a, b)+(1-\alpha) L(a, b)$, respectively.

For any $\varepsilon>0$ and $x>0$, we have

$$
\begin{aligned}
& L_{-\frac{2 \alpha+1}{3}+\varepsilon}\left((1+x)^{3}, 1\right)-\left[\alpha H\left((1+x)^{3}, 1\right)+(1-\alpha) L\left((1+x)^{3}, 1\right)\right] \\
= & \frac{J(x)}{3\left[1+(1+x)^{3 \varepsilon-1-2 \alpha}\right]\left[1+(1+x)^{3}\right] \log (1+x)}
\end{aligned}
$$

and

$$
\begin{aligned}
& \lim _{x \rightarrow+\infty}\left[\alpha H(x, 1)+(1-\alpha) L(x, 1)-L_{-\varepsilon}(x, 1)\right] \\
= & \lim _{x \rightarrow+\infty}\left[\frac{2 \alpha x}{1+x}+\frac{(1-\alpha)(x-1)}{\log x}-\frac{x^{1-\varepsilon}+1}{x^{-\varepsilon}+1}\right] \\
= & +\infty
\end{aligned}
$$


where $J(x)=3\left[1+(1+x)^{2+3 \varepsilon-2 \alpha}\right]\left[1+(1+x)^{3}\right] \log (1+x)-6 \alpha(1+x)^{3}[1+$ $\left.(1+x)^{3 \varepsilon-1-2 \alpha}\right] \log (1+x)-(1-\alpha)\left[(1+x)^{6}-1\right]\left[1+(1+x)^{3 \varepsilon-1-2 \alpha}\right]$.

Let $x \rightarrow 0$, making use of the Taylor expansion, one has

$$
\begin{aligned}
J(x)= & 3 x\left[2+(2+3 \varepsilon-2 \alpha) x+\frac{1}{2}(2+3 \varepsilon-2 \alpha)(1+3 \varepsilon-2 \alpha) x^{2}\right. \\
& \left.+o\left(x^{2}\right)\right]\left[2+3 x+3 x^{2}+o\left(x^{2}\right)\right]\left[1-\frac{1}{2} x+\frac{1}{3} x^{2}+o\left(x^{2}\right)\right] \\
& -6 \alpha x\left[1+3 x+3 x^{2}+o\left(x^{2}\right)\right]\left[2+(3 \varepsilon-1-2 \alpha) x+\frac{1}{2}\right. \\
& \left.\times(3 \varepsilon-2 \alpha-1)(3 \varepsilon-2 \alpha-2) x^{2}+o\left(x^{2}\right)\right]\left[1-\frac{1}{2} x+\frac{1}{3} x^{2}\right. \\
& \left.+o\left(x^{2}\right)\right]-(1-\alpha) x\left[6+15 x+20 x^{2}+o\left(x^{2}\right)\right][2+(3 \varepsilon-1 \\
& \left.-2 \alpha) x+\frac{1}{2}(3 \varepsilon-2 \alpha-1)(3 \varepsilon-2 \alpha-2) x^{2}+o\left(x^{2}\right)\right] \\
= & 27 \varepsilon x^{3}+o\left(x^{3}\right) .
\end{aligned}
$$

Equations (3.30)-(3.32) imply that for any $\varepsilon>0$ there exist $\delta=\delta(\varepsilon)>0$ and $X=X(\varepsilon)>1$ such that $\alpha H\left((1+x)^{3}, 1\right)+(1-\alpha) L\left((1+x)^{3}, 1\right)<$ $L_{-\frac{2 \alpha+1}{3}+\varepsilon}\left((1+x)^{3}, 1\right)$ for $x \in(0, \delta)$ and $L_{-\varepsilon}(x, 1)<\alpha H(x, 1)+(1-\alpha) L(x, 1)$ for $x \in(X, \infty)$.

\section{References}

[1] M. K. Wang, Y. M. Chu and G. D. Wang, A sharp double inequality between the Lehmer and arithmetic-geometric means, Pac. J. Appl. Math., 4(2012), no. 1, 1-25.

[2] Y. M. Chu, M. K. Wang and Y. F. Qiu, Optimal Lehmer mean bounds for the geometric and arithmetic combinations of arithmetic and Seiffert means, Azerb. J. Math., 2(2012), no. 1, 3-9.

[3] Y. M. Chu and M. K. Wang, Optimal Lehmer mean bounds for the Toader mean, Results Math., 61(2012), no. 3-4, 223-229.

[4] M. K. Wang, Y. M. Chu and G. D. Wang, A sharp double inequality between the Lehmer and arithmetic-geometric means, Pac. J. Appl. Math., 3(2011), no. 4, 281-286.

[5] Y. F. Qiu, M. K. Wang, Y. M. Chu and G. D. Wang, Two sharp inequalities for Lehmer mean, identric mean and logarithmic mean, J. Math. Inequal., 5(2011), no. 3, 301-306.

[6] M. K. Wang, Y. F. Qiu and Y. M. Chu, Sharp bounds for Seiffert means in terms of Lehmer means, J. Math. Inequal., 4(2010), no. 4, 581-586. 
[7] W. F. Xia and Y. M. Chu, The Schur harmonic convexity of Lehmer means, Int. Math. Forum, 4(2009), no. 41-44, 2009-2015.

[8] S. Toader and G. Toader, Complementaries of Greek means with respect to Lehmer means, Automat. Comput. Appl. Math., 15(2006), no. 2, 315320 .

[9] I. Costin and G. Toader, Generalized inverses of Lehmer means, Automat. Comput. Appl. Math., 14(2005), no. 1, 111-117.

[10] S. R. Wassell, Rediscovering a family of means, Math. Intelligencer, 24(2002), no. 2, 58-65.

[11] Zh. Liu, Remark on inequalities between Hölder and Lehmer means, J. Math. Anal. Appl., 247(2000), no. 1, 309-313.

[12] K. B. Stolarsky, Hölder means, Lehmer means, and $x^{-1} \log \cosh x$, J. Math. Anal. Appl., 202(1996), no. 3, 810-818.

[13] H. Alzer, Bestmögliche Abschätzungen für spezielle Mittelwerte, Zb. Rad. Prirod. -Mat. Fak. Ser. Mat., 23(1993), no. 1, 331-346.

[14] H. Alzer, Über Lehmers Mittelwertfamilie, Elem. Math., 43(1988), no. 2, $50-54$.

[15] H. N. Hu, S. S. Wang and Y. M. Chu, Optimal upper power mean bound for the convex combination of Harmonic and logarithmic means, Pac. J. Appl. Math., 4(2012), no. 1, 35-44.

[16] Y. M. Li, B. Y. Long and Y. M. Chu, Sharp bounds for the NeumanSándor mean in terms of generalized logarithmic mean, J. Math. Inequal., 6(2012), no. 4, 567-577.

[17] Y. M. Chu, M. K. Wang and Z. K. Wang, Best possible inequalities among harmonic, geometric, logarithmic and Seiffert means, Math. Inequal. Appl., 15(2012), no. 2, 412-422.

[18] Y. M. Chu, S. W. Shou and W. M. Gong, Inequalities between logarithmic, harmonic, arithmetic and centroidal means, J. Math. Appl., 2(2011), no. $2,1-5$.

[19] Y. M. Chu and M. K. Wang, Optimal inequalities between harmonic, geometric, logarithmic, and arithmetic-geometric means, J. Appl. Math., 2011(2011), Article ID 618929, 9 pages. 
[20] Y. M. Chu, S. S. Wang and C. Zong, Optimal lower power mean bound for convex combination of harmonic and logarithmic means, Abstr. Appl. Anal., 2011(2011), Article ID 520648, 9 pages.

[21] W. F. Xia and Y. M. Chu, Optimal inequalities related to the logarithmic, identric, arithmetic and harmonic means, Rev. Anal. Numér. Théor. Approx., 39(2010), no. 2, 176-183.

[22] W. F. Xia, Y. M. Chu and G. D. Wang, The optimal upper and lower power mean bounds for a convex combination of the arithmetic and logarithmic means, Abstr. Appl. Anal., 2010(2010), Article ID 604804, 9 pages.

[23] Y. M. Chu and W. F. Xia, Two optimal double inequalities between power mean and logarithmic mean, Comput. Math. Appl., 60(2011), no. 1, 83-89.

[24] B. C. Carlson, The logarithmic mean, Amer. Math. Monthly, 79(1972), 615-618.

[25] T. P. Lin, The power mean and the logarithmic mean, Amer. Math. Monthly, 81(1974), 879-883.

[26] H. Alzer, Ungleichungen für Mittelwerte, Arch. Math., 47(1986), no. 5, 422-426.

[27] F. Burk, Notes: The geometric, logarithmic, and arithmetic mean Inequality, Amer. Math. Monthly, 94(1987), no. 6, 527-528.

[28] J. Sándor, On the identric and logarithmic means, Aequations Math., 40(1990), no. 2-3, 261-270.

[29] G. Allasia, C. Giordano and J. Pečarić, On the arithmetic and logarithmic means with applications to stirling's formula, Atti Sem. Mat. Fis. Univ. Modena, 47(1999), no. 2, 441-455.

[30] H. Alzer and S. L. Qiu, Inequalities for means in two variables, Arch. Math., 80(2003), no. 2, 201-215.

[31] P. Kahlig and J. Matkowski, Functional equations involving the logarithmic mean, Z. Angew. Math. Mech., 76(1996), no. 7, 385-390.

[32] A. O. Pittenger, The logarithmic mean in $n$ variables, Amer. Math. Monthly, 92(1985), no. 2, 99-104.

[33] G. Polya and G. Szegö, Isoperimetric Inequalities in Mathematical physics, Princeton University Press, Princeton, 1951. 
[34] E. B. Leach and M. C. Sholander, Extended mean values II, J. Math. Anal. Appl., 92(1983), no. 1, 207-223.

[35] J. Sándor, A note on some inequalities for means, Arch. Math., 56(1991), no. 5, 471-473.

[36] H. Alzer, Ungleichungen für $(e / a)^{a}(b / e)^{b}$, Elem. Math., 40(1985), 120123.

[37] A. O. Pittenger, Inequalities between arithmetic and logarithmic means, Univ. Beograd. Publ. Elektrotehn. Fak. Ser. Mat. Fiz., (1980), no. 678715, 15-18.

[38] A. O. Pittenger, The symmetric, logarithmic and power means, Univ. Beograd. Publ. Elektrotehn. Fak. Ser. Mat. Fiz., (1980), no. 678-715, 1923.

[39] K. B. Stolarsky, The power and generalized logarithmic means, Amer. Math. Monthly, 87(1980), no. 7, 545-548.

\section{Received: July 5, 2013}

